\title{
RECHERCHES SUR L'ÉTIOLOGIE \\ DE LA DISTOMATOSE HÉPATIQUE AU TONKIN
}

\author{
Par H. GALLIARD
}

L'étiologie de la distomatose hépatique due à Clonorchis sinensis a été étudiée au Japon et en Chine par différents auteurs. Mais il semble qu'une certaine confusion ait régné quant à l'identité exacte des formes évolutives, cercaires et métacercaires, de ce parasite. D'autre part, certains mollusques et poissons susceptibles de servir d'hôtes intermédiaires appartiennent, au Tonkin, à des espèces différentes de celles qui ont été trouvées dans les autres pays. Il nous a donc semblé utile de reprendre la question et de chercher à déterminer les causes de l'infestation.

$$
* *
$$

La distomatose à Clonorchis sinensis est connue en Indochine depuis 1887, époque où Grall la découvrit chez des Annamites du Tonkin. Depuis lors, le parasite a été retrouvé par tous les observateurs. C'est surtout en 1903 et 1904 que sa fréquence au Tonkin a été bien mise en évidence par une note de Gaide sur l'étude clinique et anatomo-pathologique.

En 1911, Mathis et Léger, au cours de leurs recherches sur le parasitisme intestinal au Tonkin, avaient été frappés de la fréquence du parasite dans le delta tonkinois. Ils avaient constaté que la moyenne générale des parasites était de 40 p. 100. Par province, ils trouvaient: Hadong 73 p. 100 , Hanam, 56 p. 100, Namdinh 53 p. 100 , Phulangthuong 40 p. 100 . Par contre, la proportion des parasités n'est que de 3 à 4 pour 100 dans la Haute Région.

A ce propos, ces auteurs se sont élevés contre l'opinion généralement admise que la douve de Chine est, dans le Delta, moins fréquente que dans les autres régions du Tonkin et ont démontré que les divers observateurs avaient été induits en erreur et que les individus examinés avaient séjourné dans différentes localités du Tonkin. Le soin qu'ils ont pris eux-mêmes de n'examiner que les

Annales de Parasitologie, T. XVII, $\mathrm{N}^{\circ} 3 .-1^{\text {er }}$ mai 1939 , p. 236-244. 
individus n'ayant jamais quitté les environs immédiats de la région où ils étaient nés, leur a permis de préciser l'aire de distribution.

C'est pour cela que les statistiques d'hôpitaux ne donnent pas de résultats faciles à interpréter. Ainsi les statistiques faites à l'hôpital du Protectorat à Hanoï ont donné des pourcentages beaucoup plus faibles que ceux obtenus par Mathis et Léger : Mouzels 18,7 p. 100 en $1907,9,66$ p. 100 en 1908, Le Roy des Barres 1,8 p. 100, 4,1 p. 100,4 p. 100,8 p. 100 de 1911 à 1915 , Polidori 3,9 p. 100 en 1921. Nous-même avec Ngu et Quat en 1937, avons trouvé 8,16 p. 100 sur 500 examens.

Par contre, à Hanoï également, à l'Hôpital Militaire de Lanessan, les statistiques donnent des résultats entièrement différents. Seguin a trouvé une proportion de 50 p. 100 d'individus parasités, Mathis et Léger ont trouvé 27,92 p. 100.

Ces résultats ne nous renseignent certainement pas d'une façon exacte sur la proportion vraie des distomés, et on ne peut en tirer aucune conclusion quant aux variations du taux de l'infestation chez l'homme au Tonkin dans le cours des 25 dernières années.

C'est donc au Tonkin que l'infestation humaine semble localisée. En Cochinchine, Noc (1908) ne l'a jamais observée. Les recherches de Mathis et Léger avaient porté également sur le Nord-Annam où ils n'avaient trouvé qu'une proportion de $3 \mathrm{p}$. 100. Le $\mathrm{D}^{r}$ Chesneau, de Thanh-Hoa, a eu l'amabilité de me communiquer les résultats de ses examens effectués en 1935, 1936 et 1937. Les chiffres trouvés ont été respectivement : 0,4 p. 100 (sur 951 examens), 0,4 p. 100 (sur 1.172 examens) et 0,8 p. 100 (sur 1.120 examens). C'est dire qu'en Annam, l'infestation est exceptionnelle chez l'homme.

Notons également que Clonorchis sinensis n'est pas le seul distome parasitant le foie de l'homme en Indochine. Bédier et Chesneau (1929) ont trouvé en effet au Laos, à Vientiane et Thakkek, dans trois autopsies, des formes adultes qu'ils ont rapportées à Opisthorchis felineus, ou peut-être Opisthorchis viverrini qui a été signalé au Siam, dont le Laos n'est séparé que par la vallée du Mékong. D'après ces auteurs, cette distomatose serait assez fréquente au Moyen Laos et semble y exister à l'exclusion de toute autre. A Thakkek, on trouve un pourcentage de 23 p. 100, à Vientiane 15,48 p. 100 , d'après l'examen des œufs.

$$
* *
$$

Mathis et Léger, dès 1911, avaient essayé de résoudre le problème de l'étiologie de la distomatose hépatique au Tonkin. Ils 
avaient fait divers essais de transmission avec des mollusques infectés au contact de selles riches en œufs. Malgré les résultats négatifs de leurs recherches, ils avaient obtenu la conviction que " la solution du problème étiologique devrait être cherchée dans les essais de transmission aux animaux par les aliments que consomment les indigènes du Delta ».

En 1911, Kobayashi avait démontré que 13 poissons d'eau douce pouvaient jouer le rôle d'hôte intermédiaire. Muto, en 1918, au Japon, découvrit le premier hôte intermédiaire et démontra que le miracidium de Clonorchis évoluait chez Bithynia striatula var. japonica. En Chine le cycle évolutif a été étudié par E.-C. Faust et O.-K. Khaw. En 1935, Yamaguti a réalisé à nouveau le cycle évolutif et obtenu une cercaire différente de celle qui avait été décrite par les auteurs précédents.

Faust (1925) a montré que Bithynia striatula existe dans toute la Chine et que sa fréquence correspondait à celle du parasitisme par les douves ; mais elle est absente dans le Nord-Ouest de la Chine. Cependant, d'après Faust et Khaw (1927), elle n'a jamais été trouvée infectée même dans les mares où existent des poissons porteurs de métacercaires. Bithynia fuchsiana est l'hôte le plus important dans le Nord de la Chine. $B$. longicornis semble jouer un rôle moindre. Quant à Melania hongkongensis, c'est un hôte intermédiaire efficient dans certaines conditions.

Hsu et Chow en 1935 ont repris les recherches concernant les hôtes intermédiaires dans la région de Canton. Ils n'ont trouvé aucune cercaire chez 190 spécimens de Bithynia longicornis disséqués, et un seul spécimen de Parafossalurus striatulus infecté sur 470 .

Au Tonkin, où la distomatose est confinée, comme elle est confinée en Chine, mais moins étroitement, dans la province du Kwantung (Canton et Swatow), nous avons recherché quels pouvaient être les hôtes intermédiaires de Clonorchis sinensis et quelles étaient les raisons de la disproportion entre le taux des infestations signalée par Mathis et Léger dans les diverses régions du Tonkin.

Notons que Mathis et Léger avaient fait une étude de la répartition géographique des mollusques au Tonkin, et ils avaient attiré l'attention sur deux espèces de Paludina (Vivipara). "L'inégalité de répartition pour ces deux derniers gastropodes (qui sont fréquents dans le Delta et rares dans la Moyenne et Haute Région) concorde dans l'ensemble avec la distribution différente de la distomatose hépatique au Tonkin. Nous supposons que l'une au moins de ces deux paludines doit servir d'hôte intermédiaire à Clonorchis 
sinensis ». Ces auteurs étaient certainement mal informés en ce qui concerne les Vivipara ou tout au moins l'une d'elles qui est le mollusque le plus commun dans tout le Tonkin. D'autre part, il est curieux de constater que la présence de mollusques du genre Bithynia, si abondants dans le Delta, ne leur ait jamais été signalée.

Nos investigations, faites aussi bien dans le Delta que dans la Haute Région, ont porté sur les différentes espèces de mollusques qui s'y rencontrent, et particulièrement les Bithynia et les Melania dont on connaît le rôle dans les autres régions.

$$
* *
$$

Nous avons rencontré deux espèces de Bithynia, B. longicornis et $\dot{B}$. chaperi. Les deux espèces sont parfois mélangées dans les mêmes gîtes. La première est relativement rare et c'est la seconde qui doit constituer le principal hôte intermédiaire de Clonorchis sinensis.

Ces espèces se rencontrent dans des gîtes divers, principalement dans les rizières (fig.), en toute saison, mais particulièrement au printemps. Nous ne les avons pas rencontrées ailleurs que dans la zone basse du Delta. Dès que l'on arrive dans la Moyenne Région, elles sont remplacées surtout par des Planorbis et diverses Melania.

Nous avons trouvé deux espèces de Melania, M. variabilis et M. tuberculata. M. tuberculata est très commune dans le Delta, dans les eaux stagnantes ou faiblement courantes, alors que $M$. variabilis se rencontre non seulement dans les régions d'altitude, comme on l'a signalé, mais aussi dans les localités peu élevées, à condition que l'eau soit suffisamment courante et claire. M. tuberculata est d'ailleurs moins spécifique en ce qui concerne ses gìtes et son aire de distribution empiète fréquemment sur celle de l'autre espèce.

Parmi les autres espèces communes, nous avons trouvé deux espèces de Vivipara, $V$. polyzonata et $V$. quadrata, dans toutes les régions du Tonkin, mais bien que ces espèces soient remarquablement ubiquistes, elles ne sont que très rarement parasitées par des cercaires quelconques, et leur rôle doit être pratiquement nul.

L'identification des cercaires est souvent malaisée. Faust (1930) a décrit dix espèces de cercaires chez des Bythinia dont l'une rappelant beaucoup celle de Clonorchis sinensis. Ici nous avons trouvé huit cercaires et deux métacercaires différentes chez des Bithynia, trois chez Melania, trois chez Vivipara, une chez Limnæa, aucune chez Ampullaria ni chez aucun mollusque bivalve. 
Quoi qụ’il en soit, nous avons trouvé dans deux localités du Delta, aux environs de Hanoï, des cercaires de Clonorchis chez Bithynia chaperi : un exemplaire infecté sur 15 disséqués dans la première, et trois sur 30 disséqués dans la seconde. Ce qui fait quatre fois sur plus de 600 exemplaires disséqués en tout. Chez un seul exemplaire de Melania tuberculata, nous avons trouvé également des cercaires de Clonorchis sinensis, sur plus de 250 dissections.



FIG. - Récolte des mollusques dans une rizière du delta. Gîte à Bithynia chaperi.

Ainsi les mollusques qui jouent un rôle important dans la transmission de Clonorchis sinensis au Tonkin sont Bithynia chaperi, qui est beaucoup plus commune que B. longicornis, et Melania tuberculata, et c'est certainement leur présence dans le Delta, à l'exclusion des autres régions, qui explique la localisation de la distomatose hépatique.

$$
\text { *** }
$$

Nos recherches ont également porté sur le deuxième hôte intermédiaire. 
On sait quelle confusion a régné longtemps quant à l'identité exacte de la métacercaire de Clonorchis. En se basant sur des recherches expérimentales, Hsu et Khaw (1936) ont décrit une forme qui n'a rien de commun avec la forme décrite par Kobayashi en 1917, Faust et Khaw (1).

D'autre part, nous avons cherché, par la méthode de digestion artificielle, les métacercaires chez seize espèces différentes de poissons provenant directement des rizières, mares et bassins des villages, soit des différents marchés des provinces du Delta. Nous avons trouvé des métacercaires de Clonorchis sinensis chez Culter brevicauda. Mais le nombre des poissons examinés, non plus que le nombre des chats infestés expérimentalement, n'est pas encore suffisamment élevé pour nous permettre d'en tirer des conclusions définitives. D'après nos premières recherches, il semble, pour des raisons épidémiologiques, que Carassius auratus et Anabas scandens soient, avec l'espèce précitée, les plus susceptibles de conférer l'infestation à l'homme et aux animaux.

$$
* *
$$

Il est cependant évident que l'infestation de l'homme est due à l'ingestion de poisson cru ou insuffisamment cuit hébergeant des métacercaires. La consommation de poisson cru est courante au Tonkin. Houdemer, en 1934, a attiré l'attention sur certaines pratiques en usage au Tonkin ( An goi ») et qui consistent à manger en particulière abondance du poisson cru à certaines époques de l'année. Parmi les neuf espèces de poissons citées par cet auteur comme étant le plus communément consommées, trois au moins ont été reconnues au Japon par Kobayashi comme hôtes intermédiaires hébergeant des métacercaires.

Faust (1935) a montré qu'en Chine la distribution géographique de l'infestation de l'homme ne coïncidait pas avec celle des animaux domestiques, chien et chat. L'infestation humaine et animale est considérable dans le Sud, à Canton, et dans certaines localités du Kwantung où l'indice atteint parfois $100 \mathrm{p}$. 100. Dans le centre et au Nord, où le poisson n'est pas consommé cru elle est au contraire rare chez l'homme alors que 30 p. 100 des animaux sont infestés.

(1) Signalons que nous avons trouvé une métacercaire, absolument identique à celle qui a été décrite par Hsu et Khaw, dans l'hépato-pancréas d'une Bithynia (B. longicornis) infestée avec des cercaires de Clonorchis. 
Cependant, Faust (1924) a pensé que si au Chekiang l'infestation était surtout commune chez le chat, c'était dù à ce que les métacercaires se trouvant sous les écailles des poissons, il n'y a rien d'étonnant à ce que les chats s'infestent plus facilement que l'homme qui ne consomme que le poisson écaillé. Mais des recherches récentes de $\mathrm{Hsu}$ et Chow ont montré que l'immense majorité des métacercaires de Clonorchis était logée dans les muscles.

Il est donc certain qu'en Chine le parasite, trouvant à se développer partout, la répartition géographique de l'infestation humaine est strictement conditionnée par le mode d'alimentation.

Nous avons d'ailleurs pu constater nous-même que si l'infestation humaine par Clonorchis est si rare en Annam, alors qu'elle est si fréquente au Tonkin, la seule raison en est que la consommation de poisson cru s'y fait exceptionnellement, car l'hôte intermédiaire favorable, Bithynia chaperi se trouve partout en très grande abondance.

Par contre, si au Tonkin la distomatose est surtout localisée à la région du Delta, comme l'avaient démontré Mathis et Léger, c'est dû surtout à ce que les Bithynia, et en particulier B. chaperi, n'existent qu'en très faible quantité dans la Moyenne Région et sont introuvables dans la Haute Région.

\section{RÉSUMÉ}

Les recherches sur l'étiologie de la distomatose hépatique à Clonorchis sinensis ont porté d'abord sur la distribution géographique des mollusques au Tonkin et dans une partie de l'Annam.

Au Tonkin, les espèces les plus communes sont Bithynia chaperi, B. longicornis (beaucoup plus rare), Melania tuberculata, M. variabilis, Vivipara polyzonata et $V$. quadrata. Les trois premières sont localisées à la région du Delta, $M$. variabilis existe dans la Haute Région, les deux Vivipara sont ubiquistes.

Les Bithynia sont certainement les plus réceptives à l'infestation par les formes évolutives de différents trématodes. 8 cercaires et 2 métacercaires ont été trouvées chez B. chaperi, 3 chez Melania, 3 chez Vivipara, 1 chez Limnæa.

A deux reprises des cercaires de Clonorchis sinensis ont été trouvées chez Bithynia chaperi (4 exemplaires infestés sur 600); 1 seul exemplaire de Melania tuberculata sur 250 a été trouvé infesté.

B. chaperi est donc, en raison de son abondance, certainement 
l'espèce la plus favorable à la transmission du parasite. Son absence des régions élevées du Tonkin explique la localisation de la distomatose dans la région du Delta.

De nos recherches préliminaires sur le deuxième hôte intermédiaire, soit par la recherche des métacercaires chez 16 espèces différentes de poissons, soit par l'infestation des chats et des chiens, nous ne pouvons encore tirer des conclusions définitives. Nous avons trouvé des métacercaires chez Culter brevicauda. Les autres espèces qui, provisoirement, pour des raisons épidémiques, nous paraissent responsables de la contamination humaine et' animale, sont Carassius auratus, l'hôte classique, et Anabas scandens.

Les habitudes alimentaires consistant à manger du poisson cru expliquent la distribution géographique de l'infestation qui est commune au Tonkin, mais rare au Nord de l'Annam, où cependant B. chaperi pullule. Par contre, au Tonkin, cette répartition est étroitement conditionnée par celle des mollusques, $B$. chaperi hôte intermédiaire certain et Melania tuberculata, hôte intermédiaire possible.

\section{Bibliographie}

FaUst (E. C.). - The epidemiology of Cionorchis infection in China. Far east. Assoc. Trop. Med. Trans. Sixth Biernial Congress, Tokyo, I, 1925, p. 397.

Larval flukes associated with the cercarix of Clonorchis sinensis in Bithynoid snails in China and adjacent territory. Parasitology, XXII, 1930 , p. 145.

A preliminary note on the life history of Clonorchis sinensis in Chekiang province, China. Amer. Jl. Hyg., IV, 1924, p. 69.

Faust (E. C.) et Kraw (О. К.). - Studies on Clonorchis sinensis. Amer. Jl. of Hyg. Monogr. ser., $\mathrm{n}^{\circ}$ 8, 1927.

Game. - De la distomatose hépatique au Tonkin. Ann. Méd. el Hyg. coloniales, 1905 , p. 560.

Galliard (H.), NGu (D. V.) et Quat (P. H.). - Recherches sur le parasitisme intestinal à Hanoï. Bull. Soc. Méd. Chir. Indochire, XV, 1937.

Grall. - Deux observations de douves chez l'homme. Arch. de Méd. navale, 1887 , p. 459.

Houdemer (E.). - Au sujet d'une coutume favorisant l'infestation des Indochinois par Clonorchis sir.ensis. Bull. Soc. Path. exot., XXVII, 1934, p. 21.

Hsu (Н. F.) et Chow (C. Y.). - Studies on certain problems of Clonorchis sinensis. II. Investigation in the endemic center of China, the Canton area. Chin. Med. Journ., LI, 1937, p. 341.

Hsu (H. F.) et Khaw (O. K.). - On the cysts and second intermediate hosts of Clonorchis sinensis in the Peiping area. Chin. Med. Journ., L, 1936, p. 1609. 
KoвaYashi (H.). - On the life history and morphology of the liver distome (Clonorchis sinensis). Mitteil. Medez. Fachschule zu Keijo, I, 1917, p. 1917.

MATHIS (C.) et LÉGER (M.). - Recherches de parasitologie et de pathologie humaines et animales au Tonkin. Masson et Cie, Paris, 1911.

Mute (M.). - Ueber den ersten Zwischenwirt von Clonorchis sinensis. Verhandl. Jap. Path. Gesell. Tokyo, VIII, 1918, p. 151.

Nagano (K.). - Studies on the Problems of Clonorchis sinensis. Far East. Assoc. Trop. Med. Trans. Sixth Biennial Corgress, Tokyo, I, 1925, p. 379.

YAMaguti (S.). - Ueber di cercariæ von Clororchis sinensis. Zeitschr. f. Parasit., VIII, 1935 , p. 183.

Laboratoire de Parasitologie de l'Ecole de médecine de Hanoï (Indochine)

\section{EXPLICATION DES PLANCHES XV et XVI}

FIG. 1. - Rizière du delta. Gite à Bithynia chaperi et Melania tuberculata. Les mollusques se trouvent, soit dans la boue, entre les pieds de riz, soit sur les herbes du talus.

FIG. 2. - Mare dans un village du delta. Gìte à Bithynia longicornis et Vivipara polyzonata.

Fıg. 3. - Puits à l'entrée d'un village du delta. Gite à Vivipara. L'eaù sert aux usages domestiques; elle est à l'abri de toute pollution d'origine humaine.

FIg. 4. - Ces puits contiennent une végétation abondante : Lotus, Pistia, Salvinia, et servent de viviers à poissons. Rares mollusques, le plus souvent Vivipara, très rarement Bithynia. 
ANNALES DE PARASITOLOGIE

T. XVII, No 3, 1er Mai 1939
Planche XV

(Mémoire Galliard)
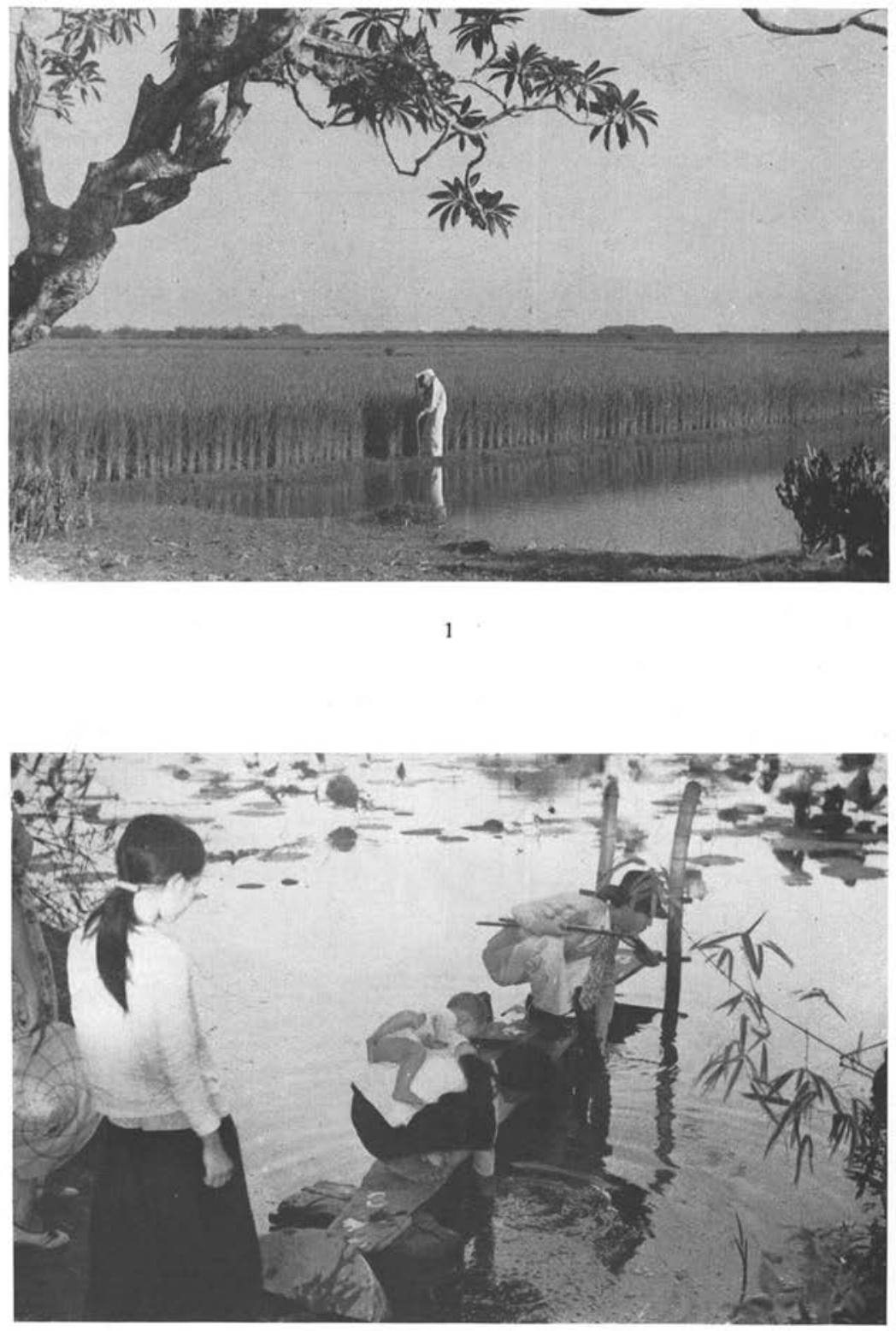



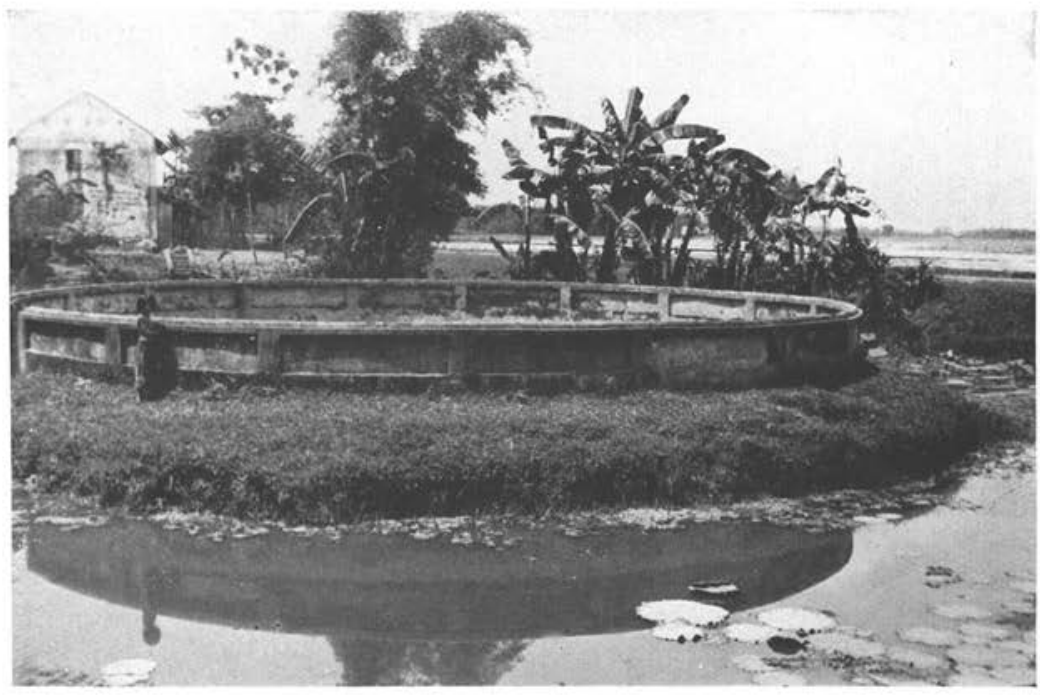

3




